\title{
Nuclear phenotypes of Triatoma infestans Malpighian tubules cultivated in vitro in presence of sodium valproate
}

\section{Alessandra Bassani*, Vera L. C. C. Rodrigues, Maria Luiza S. Mello}

\begin{abstract}
Malpighian tubules of $T$. infestans cultivated in vitro in presence of $0.05 \mathrm{mM}$ sodium valproate (VPA) for $1-4 \mathrm{~h}$ had their nuclear phenotypes investigated regarding induction of chromatin remodeling. Under present conditions, although a few nuclei demonstrated heterochromatin decondensation, no overall differences resulted in comparison to tests in which live insects are injected with VPA.
\end{abstract}

\section{Key words:}

Chromatin, epigenetics, valproic acid

\section{Introduction}

Valproic acid/sodium valproate (VPA), an anticonvulsant widely prescribed for treatment of seizure disorders, is a well-known histone deacetylase inhibitor and a chromatin remodeling agent ${ }^{1,2}$. However, in specimens of Triatoma infestans injected with VPA solutions, the response of the Malpighian tubule cells regarding chromatin decondensation is variable ${ }^{3}$.

Considering the hypothesis that the drugs injection may not result totally effective to reach the cells equally, in this work we examined the nuclear phenotypes of $T$. infestans Malpighian tubules cultivated in vitro in presence of VPA.

\section{Results and Discussion}

Malpighian tubules removed from 5th instar nymphs of T. infestans (Klug) supplied by SUCEN (Mogi-Guaçu, SP) were cultivated for 1 to $4 \mathrm{~h}$ in the Leibovitz L-15 medium (Sigma, St. Louis, USA) plus 10\% tryptose phosphate broth (Sigma) and $10 \%$ inactivated fetal calf serum at $29^{\circ} \mathrm{C}$, according to unpublished data by M. Pudney and M. G. R. Verma ${ }^{4}$ in presence or absence of $0.05 \mathrm{mM}$ VPA (Sigma). Then, they were subjected to the Feulgen reaction $^{5}$ and examined using Axiophot 2 photomicroscope (Carl Zeiss, Oberkochen, Germany).

The nuclear phenotypes identified in Malpighian tubule cells of $T$. infestans usually contain one or several heterochromatin bodies (Fig. 1). Nuclei with heterochromatin decondensation are rare. When cultivated in presence of $0.05 \mathrm{mM}$ VPA the Malpighian tubule cells did not exhibit change in frequency of their nuclear phenotypes, except under a 4-h-treatment. In this case, there was apparently a slightly increased frequency of nuclei with heterochromatin decondensation (Fig. 2). This result is similar to previously reported for $T$. infestans specimens injected with the drug $^{3}$.
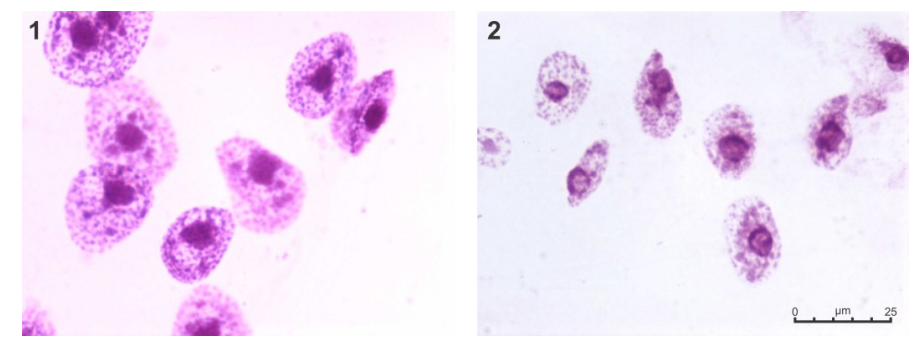

Fig. 1. Untreated control Malpighian tubule cell nuclei of T. infestans. Fig. 2. Malpighian tubule cell nuclei of $T$. infestans after treatment with $0.05 \mathrm{mM}$ VPA for $4 \mathrm{~h}$. Scale bar $=25 \mu \mathrm{m}$.

\section{Conclusions}

Under present experimental conditions, Malpighian tubules of $T$. infestans cultivated in vitro respond to VPA treatment with only a few nuclei undergoing chromatin remodeling, and not differing from findings in which the drug was injected into the insect.

Longer organ culture periods and higher VPA doses are still required to demonstrate whether there is some differential efficiency of organ cultivation in presence of VPA in comparison to the drug injection into the insect, with a view to promoting an epigenetic effect on chromatin.

\section{Acknowledgement}

Financial support: FAPESP (grants no. 2015/10356-2 and 2018/07888-0); CNPq (grant no. 304668/2014-1)

\footnotetext{
${ }^{1}$ Göttlicher, M. et al. Embo J. 2001, 20, 6969.

${ }^{2}$ Phiel, C. J. et al. J. Biol. Chem. 2001, 276, 36734.

${ }^{3}$ Alvarenga, E. M. et al. Acta Histochem. 2016, 118, 401.

${ }^{4}$ Hink, W. F. A Compilation of Invertebrate Cell Lines and Culture Media. In Invertebrate Tissue Culture (K. Maramorosch, ed.). Acad. Press 1976.

${ }^{5}$ Mello, M. L. S., B. C. Acta Histochem. 2017, 119, 603.
} 